\title{
Concordance between body fat percentage stablished by bio impedance and estimation formulas based in anthropometric measurements
}

\begin{abstract}
Obesity is one of the most important health issues worldwide. According to the Mexican National Survey of Health and Nutrition 2018 "ENSANUT", 76.8\% of women and 73\% of men who are 18 years or older are overweight or obese, and the majority are not well diagnosed. One of the greatest difficulties in order to diagnose this disease is the poor correlation between the BMI and the real fat percentage.

There are different ways to estimate the fat percentage. Some of them are the formulas based on anthropometric measures. We conducted a retrolective, descriptive, transversal trial to analyze the correlation between the fat percentage measured by bioelectrical impedance with In Body 230 and the one calculated with the Deurenberg, Lean, and CUN BAE formulas in Mexican adults.

We analyzed 319 people, 111 men, and 208 women. The average age was 43.8 , and 44.13 years, respectively. The average BMI for each gender was $36.5 \mathrm{~kg} / \mathrm{m} 2$ and $33.8 \mathrm{~kg} / \mathrm{m} 2$, and the average fat percentage was 38.23 and 45.53 respectively. We calculated de Pearson correlation index between fat percentage mesure by bioelectrical impedance and the one calculated by the formulas enlisted before. We also calculated the $\mathrm{W} / \mathrm{H}$ and the average between the three formulas.

The Pearson correlation index for Deurenberg's formula was 0.71 , for men and 0.816 for women. For Lean's formula was 0.622 for men and 0.701 for women, and for CUN BAE's formula 0.77 for men, and 0.856 for women. All of them with estatistical significance $(\mathrm{p}<0.001)$. For the $\mathrm{W} / \mathrm{H}$ was $0.702(\mathrm{p}<0.001)$ for men and $0.681(\mathrm{p}<0.001)$ for women, and for the average of the three formulas was $0.73(\mathrm{p}<0.001)$ for men and $0.828(\mathrm{p}<0.001)$ for women.

In conclusion CUN BAE's and Deurenberg's formulas have a higher correlation with the fat percentage in both genders, and CUN BAE's formula is the one with more correlation in women.
\end{abstract}

Keywords: obesity, bio impedance, fat porcentaje, adiposity

\author{
Volume II Issue 5 - 202I
}

\author{
Llamosas-Sentíes Regina,' Martínez Borja \\ Livia M, ${ }^{2}$ Ayala San Pedro J Alejandro ${ }^{2}$ \\ 'Internal Medicine Residency Department, Universidad \\ Aunónoma de México, Spanish Hospital of Mexico, Mexico \\ ${ }^{2}$ Division of Endocrinology-Internal Medicine of the Obesity and \\ Overweight Clinic, Spanish Hospital, Mexico
}

Correspondence: Livia Magdalena Martínez Borja. Obesity and Overweight Clinic, Spanish Hospital of Mexico. Ejército Nacional 613 Int 403, Granada, Miguel Hidalgo, CDMX 1 I520, Mexico,Tel 52549843,Email clinica.oobesidad@hespanol.com

Received: September 03, 202 I | Published: October II, 202 I
Abbreviations: ENSANUT, Mexican National survey of health and nutrition 2018; BMI, body mass index; SD, standard deviation; WHO, World Health Organization; CUN BAE, Clinica Universidad de Navarra-Body Adiposity Estimator; TCW, Total corporal wáter; $\mathrm{W} / \mathrm{H}$, waist/height index

\section{Introduction}

The World Health Organization (WHO) defines obesity as the accumulation of excessive fat tissue that has an impact over the individual's health. It is diagnosed with a BMI $>30 \mathrm{Kg} / \mathrm{m} 2$. Mexico has the second place in adult obesity worldwide, and the first place in childhood obesity. It is estimated that $76.8 \%$ of women and $73 \%$ of men are either overweight or obese. ${ }^{1,2}$ It is estimated that obesity is responsible of a decrease of 5-20 years of life expectancy; also, it is a risk factor for the development of metabolic, cardiovascular, muscle, humoral, and neurological diseases. ${ }^{3}$

The patophysiology of this disease is complex, nevertheless, it can be understood as an unbalance between the caloric intake and the energy expenditure. The excesive caloric intake manifests as an increase in fat deposits in different organs, affecting mainly the skeletic muscle, subcutaneous tissue, and the liver. The fat deposits in obesity consist in white fat. This type of fat is composed mainly by tryglicerides. The deposits of brown fat, which is used for thermogenesis, are diminished. ${ }^{4,5}$

White fat does not only store fat, it also secretes paracrin factors with the purpose of regulate homeostasis in metabolic tissues. ${ }^{6,7}$ The fat cell has the capacity to cense the energetic demands and regulate them. When there's an excess of white fat it deposits in tissues that are not used to it. As a consequence they go through a process called hyperthrophy, which causes an inmune response and the secretion of proinflamatory citokines, which creates insulin resistance and lipotoxicity. ${ }^{8-10}$

The deposition of fat alters the secretion por adipokines and hormones causing a proinflamatory state in people with obesity. Besides, fat excess is correlated with high free fatty acids levels in the bloodstream, which contributes to insulin resistance. Other important step in order to adquire insulin resistance is the fat deposit in visceral organs such as the heart, the liver and muscle. ${ }^{10-12}$ Finally, some obese patients have an over activation of the sympathetic nervous system, which increases cardiovascular risk. ${ }^{10,13}$ 
Even though the BMI is widely used, it does not have the capacity to estimate fat percentage, neither the fat localization (visceral or subcutaneous). There are different factor such as gender, age, sex, ethnicity and limb length that are not included in the calculation of BMI, although these factors do have an impact over fat percentage. ${ }^{14-16}$

Actually, fat percentage has taken an important roll in the evaluation of nutritional state. It is classified the following way:

Lean: Men $<8 \%$, women $<15 \%$

Optimal: Men 8.1-15.9\%, women 15.1-20.9\%

Mild overweight: Men 16\%-20.9\%, women 21-25.9\%

Overweight: Men 21-24.9\%, women 26-31.9\%

Obesity: Men > or $25 \%$, women $>$ or $32 \%^{12}$

Body composition should be evaluated in hospitalized patients, and in patients with nutritional risk. ${ }^{16}$ Recently, a new nutritional state called "sarcopenic obesity" has been described. It is characterized by a normal BMI with a reduction in muscle mass and an increase in body fat. ${ }^{17,18}$ There are different indirect ways to estimate body fat percentage. Each method has advantages and disadvantages. For example, anthropometry is a simple method and has low cost, but it has low sensibility and reproducibility. It is not useful in hyperhydration or dehydration. . $^{1921}$ The bioelectric impedance calculates body composition as it measures the hydrated tissue capacity to conduct electric energy. It can be used on healthy individuals or un people with chronic disease. Measurments are standardized for age, gender, and ethnicity. It is a simple, no invasive method, and it has no interobserver variation. Nevertheless, its measures are not standardized for oncologic or hyper/dehydrated states. ${ }^{21}$

DEXA scan has become the reference method in clinical practice because it can be reproduced, but has low accessibility, requires trained personnel, uses radiation, and it is expensive, so it is not recommended for hospitalized patients..$^{20,22}$ This method is ideal in patients with chronic diseases (inflamatory bowel disease, celiac disease, chronic pulmonary obstructive disease), and in patients with osteoporosis. ${ }^{20}$

Finally, simple tomography can be useful in order to analyze body fat percentage using a regional análisis of the third lumbar vertebrae. It is accesible for hospitalized patients, but it uses X-rays, and requires a special software in order to evaluate free fat mass..$^{23,24}$ Even though there are differences between these methods, there is evidence that, in a populational scale, DEXA and bioelectric impedance have similar results, only having significant differences in people with BMI of 16$18 \mathrm{~kg} / \mathrm{m}^{2} .{ }^{24,25}$

An effort has been made in order to create formulas based in anthropometric measures to calculate body fat percentage. ${ }^{26,27}$ In 1991, Deurenberg et al made a trial comparing BMI with body fat percentage measured by densitometry. In this trial they suggest to adjust the BMI considering age and gender. They created Deurenberg's formula to estimate body fat percentage. ${ }^{28}$ This formula has been validated for different ethnicities and age groups. Today it is considered to be useful in patients with BMI of 13.9-40.9 Kg/m² and from 7-83 years old. None of these validating studies have been conducted in latin population. $^{28}$

Lean et al., ${ }^{29}$ conducted a trial in 1996 that was inspired by Deurenberg's work. In this trial they evaluated the importance of some other anthropometric measures (besides height and weight) to determine body fat percentage finding that tricipital fold and waist circumference to be the most useful, so they developed another formula that included waist circumference. This investigation was conducted in Glasgow, Scotland, and the demographic description don't detail ethnicity, but it is assumed that there was no significant percentage of latin population. ${ }^{29}$

Another formula developed in 2008 by Gómez-Ambrosi et al called "Clínica Universidad de Navarra-Body Adiposity Estimator" (CUN$\mathrm{BAE})$. It uses BMI, age, and gender to calculate body fat percentage. ${ }^{30}$ The results were compared to the ones obtained by plethismography This formula was useful to estimate body fat percentage, specially in women. ${ }^{30,31}$

There are several recent studies that evaluate the use of these formulas and BMI in diverse ethnic groups. ${ }^{32}$ Al-Gindan et al conducted a research in 2015 in order to create a new equation based un height, weight, and waist to estimate body fat percentage. They also evaluated Deurenberg's, Lean's, and CUN BAE formulas finding that in men the equations that include waist measurement have more correlation with body fat percentage measured by magnetic resonance. In women, the most accurate formulas were the ones that only considered age and BMI. ${ }^{33}$

Some studies have been conducted in order to identify differences in body composition between different ethnic groups. Fernández et al conducted a trial in 2010 comparing concordance between BMI and body fat percentage in Latin, Afroamerican, and from European descent men and women finding that body fat percentage in Latin women did not have correlation with BMI. ${ }^{34}$ Those findings correlated with the ones found in a trial conducted in 2001 that identified that Latin women have a higher body fat percentage in comparison with European women. ${ }^{35}$

In 2016, William W. Wong et al, conducted a trial in order to analyze the correlation between BMI and fat percentage measured by bioelectric impedance in Latin people finding that there is less correlation en older age men. This trial concludes that the percentage of misclassified patients increases with age. It also suggests that BMI can underestimate body fat percentage in Latin people. ${ }^{36}$

Given the previous evidence we conducted a trial in order to evaluate the correlation between body fat percentage measured by bioelectric impedance and the one estimated by Deurenberg's, Lean's, and CUN BAE formulas in Mexican adults that visit the Obesity and Overweight Clinic of the Spanish Hospital of Mexico.

\section{Methods}

We conducted a retrospective, observational, transversal trial in order to evaluate the correlation between body fat percentage measured by bioelectric impedance and the one estimated by Deurenberg's, Lean's, and CUN BAE formulas in Mexican adults that visit the Obesity and Overweight Clinic of th Spanish Hospital of Mexico from 2014 to March 2021.

We obtained the following demographic and anthropometric variables for each patient: weight, height, waist circumference, age, biologic gender. We measured, via bioelectric impedance with In Body 230 the following variables: total body water, percentage of muscle mass, weight of muscle mass measured in $\mathrm{Kg}$, visceral fat measured in $\mathrm{Kg}$, and body fat percentage. We calculated body fat percentage using the following formulas: 
-Deurenberg: $(1.2 *$ BMI $)+(0.23 *$ age $)-(10.8 *$ gender $)-5.4$, (gender $=0$ for women and 1 for men).

-Lean: Men $\quad(0.567 \mathrm{xWaist})+(0.101 \mathrm{xAge})-31.8$. Women: (0.439xWaist) $+(0.221 \times$ Age $)-9.4$

-CUN-BAE: $\quad-44,988+(0,503 \times$ age $) \quad+(10,689 \times$ gender $) \quad+(3,172$ $\times \mathrm{BMI})-\left(0,026 \times \mathrm{BMI}^{2}\right)+(0,181 \times \mathrm{BMI} \times$ gender $)-(0,02 \times \mathrm{BMI} \times$ age $)-$ $\left(0,005 \times \mathrm{BMI}^{2} \times\right.$ gender $)+\left(0,00021 \times \mathrm{BMI}^{2} \times\right.$ age $)$, men $=0$ y women $=1$.

Because of the reliability of the bioelectrical impedance, the measurement was done only once, meanwhile, the waist was measured twice. The anthropometric data were obtained form the record of the patients. We included every patient over 18 years old, excluding patients that had pacemakers or cerebral stimulating implants, patients without a limb, scoliosis, titanium prothesis, pregnant women, or with lower extremity edema or ascitis.

We analyzed our population distribution using the KolmogorovSmirnoff test, and we used SPSS software in order to analyze the correlation between the data obtained using the formulas based in anthropometric measurements and the estimated body fat percentage by bioelectric impedance using Pearson's correlation index.

\section{Results}

We analyzed 319 people, 111 men, and 208 women with average age og 43.8 years and 44.13 years respectively. The average BMI for each genders $36.5 \mathrm{~kg} / \mathrm{m}^{2}$, SD 7.2 and $33.8 \mathrm{~kg} / \mathrm{m}^{2}$, SD 7.3 , meanwhile the average fat percentage was $38.23 \%$, SD $7.87 \%$ and $45.53 \%$ SD $7.06 \%$ respectively. We obtained the average of each formula: Deurenberg: 48.5 SD 8.27 in men and 34.53 SD 9.55 in women, Lean 40.78 SD 9.9 in men and 45.52 SD 7.87 in women, and CUN BAE 37.73 SD6.43 in men and 45.73 SD 6.72 in women. Table 1 and 2 show the demographic description of the studied population. We conducted the Kolmogorov Smirnoff test to determine our population distribution obtaining a $\mathrm{p}=0.063$ for body fat percentage, so we concluded that our population had a normal distribution.

Table I Physical characteristics of the studied population (Men)

\begin{tabular}{|c|c|c|c|c|c|c|c|c|c|c|c|c|c|c|}
\hline $\begin{array}{l}\text { Age } \\
\text { (n) }\end{array}$ & Weight & Height & BMI & $\begin{array}{l}\text { Weight } \\
\text { Excess }\end{array}$ & $A C$ & $\%$ Fat & $\begin{array}{l}\mathrm{Kg} \\
\text { Fat }\end{array}$ & $\begin{array}{l}\text { Kg } \\
\text { Músc }\end{array}$ & TCW & Duren & Lean & $\begin{array}{l}\text { CUN } \\
\text { BAE }\end{array}$ & W/H & Average \\
\hline $\begin{array}{l}<20 \\
(4)\end{array}$ & $\begin{array}{l}\text { I00.3- } \\
\text { I33 } \\
(117.55 \\
\text { SD 0.05) }\end{array}$ & $\begin{array}{l}1.7-1.91 \\
(1.84 \\
\text { SD } \\
13.87)\end{array}$ & $\begin{array}{l}32.26- \\
36.5 \\
(34.74 \\
S D \\
I, 8)\end{array}$ & $\begin{array}{l}25.9- \\
42.16 \\
(33.31 \\
\text { SD 7.49) }\end{array}$ & $\begin{array}{l}116-154 \\
(126.88 \\
S D \\
18.23)\end{array}$ & $\begin{array}{l}32.3- \\
40.4 \\
(35.28 \\
\text { SD } \\
3.56)\end{array}$ & $\begin{array}{l}39.7- \\
43 \\
(41.18 \\
\text { SD } \\
I .4)\end{array}$ & $\begin{array}{l}33.2- \\
52.1 \\
(43.93 \\
\text { SD } \\
7.67)\end{array}$ & $\begin{array}{l}43.7- \\
65.7 \\
(55.78 \\
\text { SD 9.3) }\end{array}$ & $\begin{array}{l}37.44- \\
42.54 \\
(40.48 \\
\text { SD 2.I) }\end{array}$ & $\begin{array}{l}35.89- \\
57.34 \\
(41.98 \\
\text { SD } \\
10.3)\end{array}$ & $\begin{array}{l}31.66- \\
37.1 \\
(34.9 \\
S D \\
2.3)\end{array}$ & $\begin{array}{l}0.63- \\
0.83 \\
(0.69 \\
S D \\
0.08)\end{array}$ & $\begin{array}{l}36-46 \\
(39.12 \\
\text { SD 4.4) }\end{array}$ \\
\hline $\begin{array}{l}20-29 \\
(12)\end{array}$ & $\begin{array}{l}75.5- \\
156.7 \\
(124.73 \\
\text { SD } \\
24.14)\end{array}$ & $\begin{array}{l}1.69-1.9 \\
(1.78 \\
\text { SD } \\
0.07)\end{array}$ & $\begin{array}{l}23.6- \\
47.5 \\
(39.17 \\
S D \\
6.7)\end{array}$ & $\begin{array}{l}-4.28- \\
71.56 \\
(45.5 \mathrm{SD} \\
22)\end{array}$ & $\begin{array}{l}84-144.5 \\
(121.72 \\
S D \\
27.21)\end{array}$ & $\begin{array}{l}\text { I5.4- } \\
50.7 \\
(39.45 \\
\text { SD } \\
9.93)\end{array}$ & $\begin{array}{l}\text { II.6- } \\
73.4 \\
(50.64 \\
\text { SD } \\
\text { I8.27) }\end{array}$ & $\begin{array}{l}31.4- \\
53.6 \\
(42.02 \\
\text { SD } \\
5.84)\end{array}$ & $\begin{array}{l}40.8- \\
68.2 \\
(53.74 \\
\text { SD } \\
7.24)\end{array}$ & $\begin{array}{l}29.59- \\
57.35 \\
(47.3 \\
\text { SD 8.I) }\end{array}$ & $\begin{array}{l}18.76- \\
52.56 \\
(39.73 \\
\text { SD } \\
\text { I5.36) }\end{array}$ & $\begin{array}{l}19.74- \\
47.65 \\
(39.55 \\
\text { SD } \\
7.9)\end{array}$ & $\begin{array}{l}0.27- \\
0.82 \\
(0.65 \\
\text { SD } \\
0.15)\end{array}$ & $\begin{array}{l}23-5 I \\
(4 I \mathrm{SD} \\
9.27)\end{array}$ \\
\hline $\begin{array}{l}30-39 \\
(25)\end{array}$ & $\begin{array}{l}71.3- \\
221.9 \\
(121.13 \\
S D \\
36.29)\end{array}$ & $\begin{array}{l}1.67-1.9 \\
(1.76 \\
S D \\
0.06)\end{array}$ & $\begin{array}{l}24.1- \\
70.8 \\
(38.64 \\
S D \\
10.17)\end{array}$ & $\begin{array}{l}-2.36- \\
143.89 \\
(43.78 \\
\text { SD } \\
33.88)\end{array}$ & $\begin{array}{l}87-185 \\
(123.14 \\
S D \\
23.47)\end{array}$ & $\begin{array}{l}16.6- \\
53.2 \\
(34.48 \\
\text { SD } \\
9.47)\end{array}$ & $\begin{array}{l}I 5.5- \\
I I 8 . I \\
(50.3 \\
S D \\
24.4 I)\end{array}$ & $\begin{array}{l}30.9- \\
60.8 \\
(40.57 \\
\text { SD } \\
7.88)\end{array}$ & $\begin{array}{l}39.9- \\
78.6 \\
(51.98 \\
\text { SD 9.9) }\end{array}$ & $\begin{array}{l}30.42- \\
86.92 \\
(48.85 \\
\text { SD } \\
12.24)\end{array}$ & $\begin{array}{l}20.5- \\
76.33 \\
(41.48 \\
\text { SD } \\
13.29)\end{array}$ & $\begin{array}{l}20.71- \\
53.56 \\
(38.34 \\
\text { SD } \\
8.56)\end{array}$ & $\begin{array}{l}0.5 \mathrm{I}- \\
1.05 \\
(0.697 \\
S D \\
0.12)\end{array}$ & $\begin{array}{l}24-72 \\
(42.89 \\
\text { SD } \\
12.24)\end{array}$ \\
\hline $\begin{array}{l}40-49 \\
(37)\end{array}$ & $\begin{array}{l}80.6- \\
168.2 \\
(111.4 \\
\text { SD } \\
17.96)\end{array}$ & $\begin{array}{l}I .61- \\
I .87 \\
(1.75 \\
\text { SD } \\
0.067)\end{array}$ & $\begin{array}{l}28.4- \\
54.8 \\
(36.4 \\
S D \\
5.82)\end{array}$ & $\begin{array}{l}\text { II.08- } \\
88.34 \\
(34.78 \\
\text { SD I7.3) }\end{array}$ & $\begin{array}{l}99-167 \\
(120.42 \\
S D \\
15.82)\end{array}$ & $\begin{array}{l}25.7- \\
56.2 \\
(38.5 \\
S D \\
7.16)\end{array}$ & $\begin{array}{l}26.9- \\
86.6 \\
(43.26 \\
\text { SD } \\
13.48)\end{array}$ & $\begin{array}{l}26.6- \\
50.4 \\
(39.05 \\
\text { SD } \\
5.57)\end{array}$ & $\begin{array}{l}35.1-65 \\
(50.24 \\
\text { SD 6.8) }\end{array}$ & $\begin{array}{l}38.34- \\
70.71 \\
(48.56 \\
S D \\
6.99)\end{array}$ & $\begin{array}{l}29.18- \\
67.43 \\
(40.99 \\
\text { SD } \\
8.96)\end{array}$ & $\begin{array}{l}28.55- \\
52.36 \\
(37.86 \\
\text { SD } \\
5.55)\end{array}$ & $\begin{array}{l}0.54- \\
0.97 \\
(0.69 \\
S D \\
0.096)\end{array}$ & $\begin{array}{l}33-64 \\
(42.47 \\
\text { SD 6.98) }\end{array}$ \\
\hline $\begin{array}{l}50-59 \\
(14)\end{array}$ & $\begin{array}{l}79.3- \\
159.5 \\
(103.69 \\
\text { SD } \\
18.75)\end{array}$ & $\begin{array}{l}1.68-1.9 \\
(1.74 \\
S D \\
0.06)\end{array}$ & $\begin{array}{l}27.4- \\
46.3 \\
(34.26 \\
S D \\
4.5)\end{array}$ & $\begin{array}{l}7.92- \\
67.6 \\
(28.5 \mathrm{SD} \\
15.4)\end{array}$ & $\begin{array}{l}94-153 \\
(I 17.5 \mid \\
S D \\
14.45)\end{array}$ & $\begin{array}{l}23.7 I- \\
45.1 \\
(37.09 \\
\text { SD } \\
5.32)\end{array}$ & $\begin{array}{l}20.5- \\
54.1 \\
(37.52 \\
\text { SD } \\
8.68)\end{array}$ & $\begin{array}{l}30.6- \\
54.2 \\
(36.61 \\
\text { SD } \\
5.72)\end{array}$ & $\begin{array}{l}39.9- \\
69.4 \\
(47.48 \\
\text { SD } \\
7.12)\end{array}$ & $\begin{array}{l}39.92- \\
58.88 \\
(48.25 \\
\text { SD } \\
5.32)\end{array}$ & $\begin{array}{l}26.75- \\
60.2 \\
(40.3 \\
S D \\
8.16)\end{array}$ & $\begin{array}{l}28.76- \\
45.42 \\
(36.42 \\
S D \\
4.82)\end{array}$ & $\begin{array}{l}0.56- \\
0.85 \\
(0.68 \\
S D \\
0.07)\end{array}$ & $\begin{array}{l}32-55 \\
(41.67 \\
\text { SD 5.96) }\end{array}$ \\
\hline $\begin{array}{l}>60 \\
(19)\end{array}$ & $\begin{array}{l}67.1- \\
139.2 \\
(98.18 \\
\text { SD 18.6) }\end{array}$ & $\begin{array}{l}1.44- \\
1.84 \\
(1.7 \mathrm{SD} \\
0.09)\end{array}$ & $\begin{array}{l}22.4- \\
43.4 \\
(34.48 \\
S D \\
5.53)\end{array}$ & $\begin{array}{l}-7.42- \\
59.34 \\
(27.68 \\
\text { SD 16.7) }\end{array}$ & $\begin{array}{l}93.2-153 \\
(I|4,4| \\
S D \\
I 5.79)\end{array}$ & $\begin{array}{l}22.5- \\
50.9 \\
(37,95 \\
\text { SD } \\
8.32)\end{array}$ & $\begin{array}{l}7.1- \\
70.9 \\
(35.97 \\
\text { SD } \\
16.07)\end{array}$ & $\begin{array}{l}26.5- \\
39.1 \\
(33.84 \\
\text { SD } \\
4.03)\end{array}$ & $\begin{array}{l}35-50.5 \\
(44.24 \\
\text { SD } \\
4.93)\end{array}$ & $\begin{array}{l}35.51- \\
60.94 \\
(50.18 \\
S D \\
6.66)\end{array}$ & $\begin{array}{l}27.21- \\
61.21 \\
(39.47 \\
\text { SD } \\
8.94)\end{array}$ & $\begin{array}{l}22.87- \\
45.56 \\
(36.70 \\
\text { SD } \\
5.77)\end{array}$ & $\begin{array}{l}0.54- \\
0.85 \\
(0.68 \\
S D \\
0.09)\end{array}$ & $\begin{array}{l}29-56 \\
(42.15 \\
\text { SD 6.7) }\end{array}$ \\
\hline
\end{tabular}

BMI, Body mass index; SD, standard deviation;AC, abdominal circumference in centímeters; $\mathrm{Kg}$ Musc, $\mathrm{Kg}$ muscle, TCW, total corporal wáter;W/H,Waist/Height index; Duren: deurenberg formula heigh measured in meters 
Table 2 Physical characteristics of the studied population (Women)

\begin{tabular}{|c|c|c|c|c|c|c|c|c|c|c|c|c|c|c|}
\hline $\begin{array}{l}\text { Age } \\
\text { (n) }\end{array}$ & Weight & Height & BMI & $\begin{array}{l}\text { Weight } \\
\text { Excess }\end{array}$ & $A C$ & $\%$ Fat & $\begin{array}{l}\text { Kg } \\
\text { Fat }\end{array}$ & $\begin{array}{l}\text { Kg } \\
\text { Músc }\end{array}$ & TCW & Duren & Lean & $\begin{array}{l}\text { CUN } \\
\text { BAE }\end{array}$ & W/H & Average \\
\hline $\begin{array}{l}<20 \\
(6)\end{array}$ & $\begin{array}{l}80,2- \\
114,2 \\
(93,2 \mathrm{SD} \\
18,35)\end{array}$ & $\begin{array}{l}1,66- \\
1,71 \\
(1,69 \\
S D \\
0,025)\end{array}$ & $\begin{array}{l}27-42 \\
(33 \\
S D \\
7.7)\end{array}$ & $\begin{array}{l}7.39- \\
5.59 \\
(22.35 \\
\text { SD } \\
20,39)\end{array}$ & $\begin{array}{l}97-120 \\
(107 \\
S D \\
I 1,78)\end{array}$ & $\begin{array}{l}36.6- \\
52.8 \\
(44,86 \\
S D \\
8.1)\end{array}$ & $\begin{array}{l}29,3- \\
60,3 \\
(42,7 \\
S D \\
15,9)\end{array}$ & $\begin{array}{l}25,6- \\
30 \\
(27,93 \\
S D \\
2.21)\end{array}$ & $\begin{array}{l}34- \\
39,7 \\
(36,93 \\
S D \\
2,85)\end{array}$ & $\begin{array}{l}21-38 \\
(27.7 \\
\text { SD 9.3) }\end{array}$ & $\begin{array}{l}37-47 \\
(4 I .7 \\
S D \\
5.2)\end{array}$ & $\begin{array}{l}36-52 \\
(4.28 \\
S D \\
8.4)\end{array}$ & $\begin{array}{l}0.57- \\
0.72 \\
(0.635 \\
\text { SD } \\
0.08)\end{array}$ & $\begin{array}{l}32-46 \\
(37 \mathrm{SD} \\
7.6)\end{array}$ \\
\hline $\begin{array}{l}20- \\
29 \\
(31)\end{array}$ & $\begin{array}{l}56.8- \\
148.9 \\
(94.43 \\
\text { SD } \\
24.67)\end{array}$ & $\begin{array}{l}1.52- \\
1.75 \\
(1.63 \\
S D \\
0.06)\end{array}$ & $\begin{array}{l}22.4- \\
56.4 \\
(35.34 \\
\text { SD } \\
8.73)\end{array}$ & $\begin{array}{l}-6.48- \\
78.62 \\
(28.46 \\
\text { SD 23.2) }\end{array}$ & $\begin{array}{l}77.5- \\
\text { I55 } \\
(108.5 \\
\text { SD } \\
20.94)\end{array}$ & $\begin{array}{l}29- \\
56.3 \\
(45.24 \\
\text { SD } \\
8.02)\end{array}$ & $\begin{array}{l}\text { 19.7- } \\
78.1 \\
(45.22 \\
\text { SD } \\
\text { I7.23) }\end{array}$ & $\begin{array}{l}18.3- \\
41.1 \\
(28.17 \\
\text { SD } \\
5.65)\end{array}$ & $\begin{array}{l}19- \\
52.4 \\
(35.92 \\
\text { SD } \\
6.88)\end{array}$ & $\begin{array}{l}16.86- \\
57.69 \\
(32.09 \\
S D \\
10.65)\end{array}$ & $\begin{array}{l}30.81- \\
65.05 \\
(43.91 \\
\text { SD } \\
9.35)\end{array}$ & $\begin{array}{l}29.58- \\
57.36 \\
(44.99 \\
\text { SD } \\
8.29)\end{array}$ & $\begin{array}{l}0.49- \\
0.99 \\
(0.66 \\
\text { SD } \\
0.12)\end{array}$ & $\begin{array}{l}27-60 \\
(40 \mathrm{SD} \\
9.14)\end{array}$ \\
\hline $\begin{array}{l}30- \\
39 \\
(47)\end{array}$ & $\begin{array}{l}60-199.9 \\
(84.29 \\
\text { SD } \\
17.19)\end{array}$ & $\begin{array}{l}1.47- \\
1.74 \\
(1.63 \\
\text { SD } \\
0.06)\end{array}$ & $\begin{array}{l}22.2- \\
46.17 \\
(31.73 \\
S D \\
6.15)\end{array}$ & $\begin{array}{l}-7.31- \\
52.42 \\
(18.16 \\
\text { SD } \\
16.24)\end{array}$ & $\begin{array}{l}73.2- \\
119 \\
(95.33 \\
\text { SD } \\
10.84)\end{array}$ & $\begin{array}{l}25.4- \\
56.8 \\
(43.43 \\
S D \\
7.23)\end{array}$ & $\begin{array}{l}\text { I5.6- } \\
64 \\
(37.62 \\
\text { SD } \\
\text { I3.02) }\end{array}$ & $\begin{array}{l}19.3- \\
32.3 \\
(25.78 \\
\text { SD } \\
28.8)\end{array}$ & $\begin{array}{l}26.4- \\
42.3 \\
(34.19 \\
S D \\
4.04)\end{array}$ & $\begin{array}{l}|8.5|- \\
47.94 \\
(30.03 \\
S D \\
7.47)\end{array}$ & $\begin{array}{l}30.03- \\
50.58 \\
(40.28 \\
S D \\
4.8)\end{array}$ & $\begin{array}{l}30.71- \\
55.46 \\
(42.83 \\
\text { SD } \\
6.79)\end{array}$ & $\begin{array}{l}0.45- \\
0.77 \\
(0.59 \\
S D \\
0.07)\end{array}$ & $\begin{array}{l}26-50 \\
(37.72 \\
\text { SD 6.04) }\end{array}$ \\
\hline $\begin{array}{l}40- \\
49 \\
(52)\end{array}$ & & & $\begin{array}{l}23.1- \\
64.2 \\
(34.40 \\
S D \\
7.5)\end{array}$ & $\begin{array}{l}-2.35- \\
98.04 \\
(24.74 \\
\text { SD } \\
19.45)\end{array}$ & $\begin{array}{l}76-154 \\
(102.7 \\
S D \\
15.76)\end{array}$ & $\begin{array}{l}30.5- \\
79.2 \\
(45.31 \\
\text { SD } \\
7.7)\end{array}$ & $\begin{array}{l}17.6- \\
67.3 \\
(40.14 \\
S D \\
12.29)\end{array}$ & $\begin{array}{l}17.5- \\
61.6 \\
(27.39 \\
S D \\
6.54)\end{array}$ & $\begin{array}{l}23.8- \\
46 \\
(35.07 \\
\text { SD } \\
5.22)\end{array}$ & $\begin{array}{l}20.7- \\
70.27 \\
(35.26 \\
\text { SD } \\
9.05)\end{array}$ & $\begin{array}{l}33.03- \\
67.27 \\
(45.48 \\
\text { SD } \\
6.91)\end{array}$ & $\begin{array}{l}32.74- \\
56.66 \\
(45.96 \\
S D \\
6.17)\end{array}$ & $\begin{array}{l}0.47- \\
0.97 \\
(0.64 \\
\text { SD } \\
0.1)\end{array}$ & $\begin{array}{l}31-65 \\
(42.23 \\
S D 7)\end{array}$ \\
\hline $\begin{array}{l}50- \\
59 \\
(39)\end{array}$ & $\begin{array}{l}58.9- \\
168.7 \\
(89.12 \\
\text { SD } \\
21.97)\end{array}$ & $\begin{array}{l}1.51- \\
1.77 \\
(1.63 \\
S D \\
0.07)\end{array}$ & $\begin{array}{l}24.5- \\
53.8 \\
(33.43 \\
\text { SD } \\
6.73)\end{array}$ & $\begin{array}{l}-0.92- \\
90.69 \\
(23.12 \\
\text { SD } \\
19.08)\end{array}$ & $\begin{array}{l}81-155 \\
(103.24 \\
\text { SD } \\
14.33)\end{array}$ & $\begin{array}{l}29.7- \\
54.4 \\
(45.65 \\
\text { SD } \\
5.83)\end{array}$ & $\begin{array}{l}\text { I7.5- } \\
85.5 \\
(40.92 \\
\text { SD } \\
\text { I3.8) }\end{array}$ & $\begin{array}{l}3.6- \\
47.2 \\
(25.13 \\
S D \\
6.10)\end{array}$ & $\begin{array}{l}25.1- \\
62.1 \\
(34.93 \\
\text { SD } \\
7.02)\end{array}$ & $\begin{array}{l}24.7- \\
61.01 \\
(36.26 \\
S D \\
8.21)\end{array}$ & $\begin{array}{l}37.43- \\
70.8 \\
(47.78 \\
\text { SD } \\
6.43)\end{array}$ & $\begin{array}{l}36.19- \\
58.28 \\
(45.98 \\
\text { SD } \\
5.79)\end{array}$ & $\begin{array}{l}0.48- \\
0.88 \\
(0.63 \\
S D \\
0.08)\end{array}$ & $\begin{array}{l}34-63 \\
(43.34 \\
\text { SD 6.42) }\end{array}$ \\
\hline $\begin{array}{l}>60 \\
(33)\end{array}$ & $\begin{array}{l}62.5-121 \\
(85.87 \\
\text { SD 5.23) }\end{array}$ & $\begin{array}{l}1.44- \\
1.76 \\
(1.57 \\
S D \\
0.06)\end{array}$ & $\begin{array}{l}24.7- \\
54 \\
(35.3 \\
\text { SD } \\
7.14)\end{array}$ & $\begin{array}{l}-0.45- \\
59.62 \\
(24.46 \\
\text { SD } \\
\text { I5.58) }\end{array}$ & $\begin{array}{l}82.5- \\
\text { I4I } \\
(108.75 \\
\text { SD } \\
\text { I5.59) }\end{array}$ & $\begin{array}{l}37.2- \\
57.9 \\
(49.02 \\
\text { SD } \\
4.81)\end{array}$ & $\begin{array}{l}24.1- \\
62.5 \\
(42.66 \\
\text { SD } \\
\text { II.35) }\end{array}$ & $\begin{array}{l}\text { I6.7- } \\
32.8 \\
(23.61 \\
S D \\
3.67)\end{array}$ & $\begin{array}{l}23.5- \\
44.1 \\
(31.87 \\
\text { SD } \\
4.66)\end{array}$ & $\begin{array}{l}27.7- \\
64.93 \\
(41.63 \\
\text { SD } \\
8.89)\end{array}$ & $\begin{array}{l}40.5- \\
67.5 \\
(53.20 \\
\text { SD } \\
6.96)\end{array}$ & $\begin{array}{l}38.1- \\
58.88 \\
(48.51 \\
S D \\
5.42)\end{array}$ & $\begin{array}{l}0.01- \\
0.93 \\
(0.86 \\
\text { SD } \\
0.15)\end{array}$ & $\begin{array}{l}35-60 \\
(47.78 \\
\text { SD 6.56) }\end{array}$ \\
\hline
\end{tabular}

$\mathrm{BMI}$, body mass index; SD, standard deviation; $\mathrm{AC}$, abdominal circumference in centímeters; $\mathrm{Kg}$ Musc, $\mathrm{Kg}$ muscle; TCW, total corporal wáter; W/H, waist/height index; Duren, deurenberg formlua heigh measured in meters

We calculated Pearson's correlating index for each formula, and for the waist/height index and the average of the three formulas:

Deurenberg $0.714(\mathrm{p}<0.001)$ for men and $0.816(\mathrm{p}<0.001)$ for women.

Lean $0,622(\mathrm{p}<0.001)$ for men and $0.701(<0.001)$ for women.

CUN BAE $0.77(\mathrm{p}<0.001)$ for men and $0.856(\mathrm{p}<0.001)$ for women.
Waist/Height $0.702(<0.001)$ for men and $0.681(\mathrm{p}<0.001)$ for women.

Average of the three formulas $0.73(\mathrm{p}<0.001)$ for men and 0.828 $(\mathrm{p}<0.001)$ for women.

Graph 1 shows the correlation for each formula. Deurenberg's formula has more dispersion than Lean's or CUN BAE formulas. Also, BMI tends to calculate lower values than the other three formulas. Graphs $2 \& 3$ show the dispersion by gender.

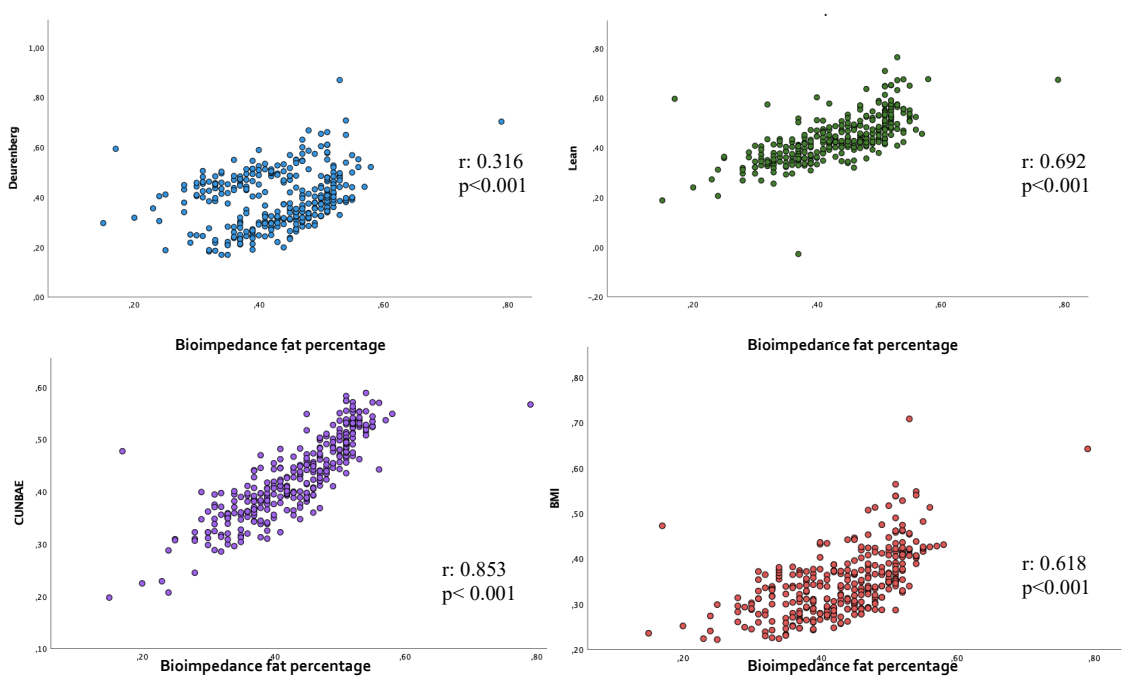

Graph I Individual formula's correlation with fat percentage.

Citation: Regina LS, Livia MBM,Alejandro ASPJ. Concordance between body fat percentage stablished by bio impedance and estimation formulas based in anthropometric measurements. Adv Obes Weight Manag Control. 202 I; I I (5):I59-I66. DOI: I0.I5406/aowmc.202I.I I.0035 I 


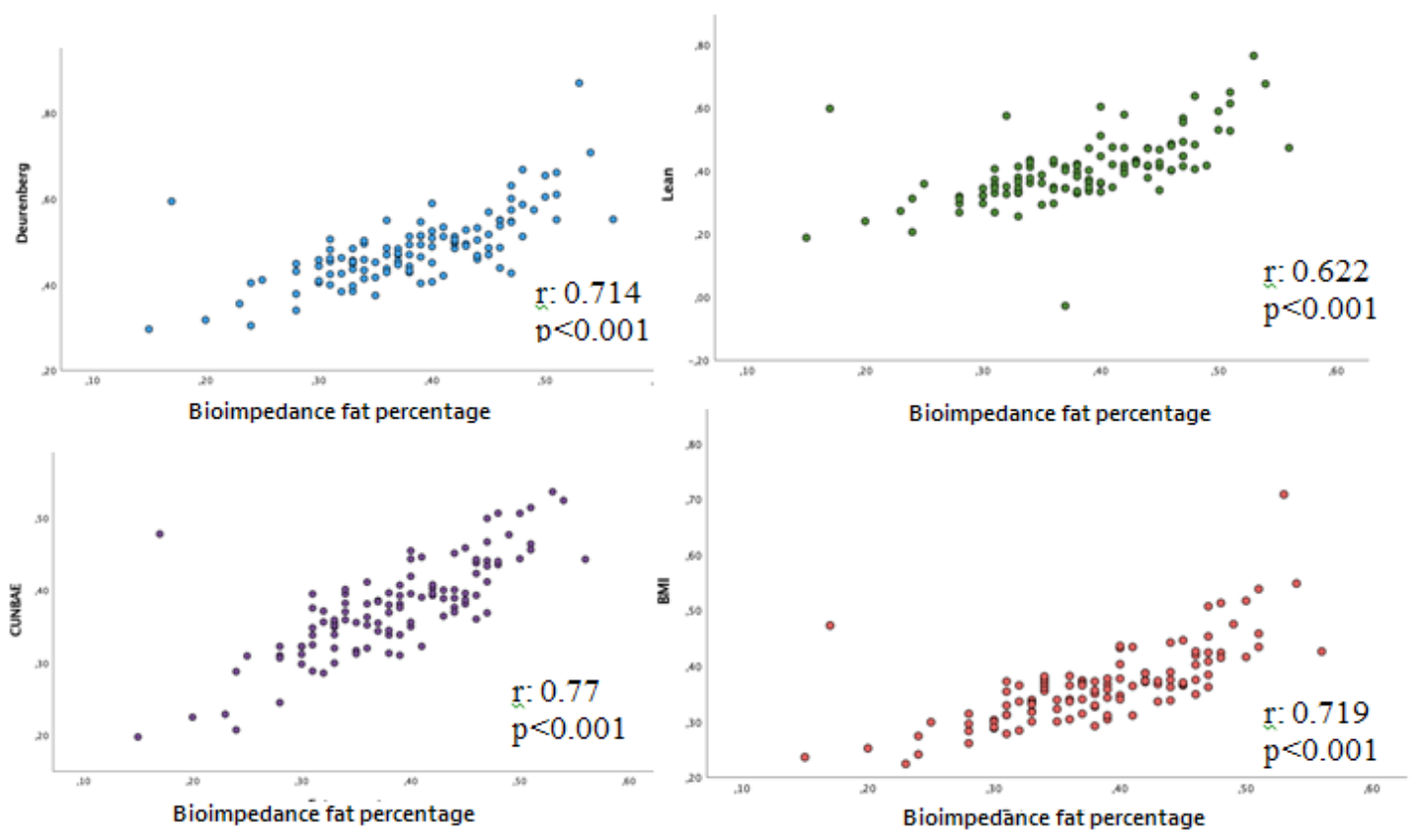

Graph 2 Correlation in men.
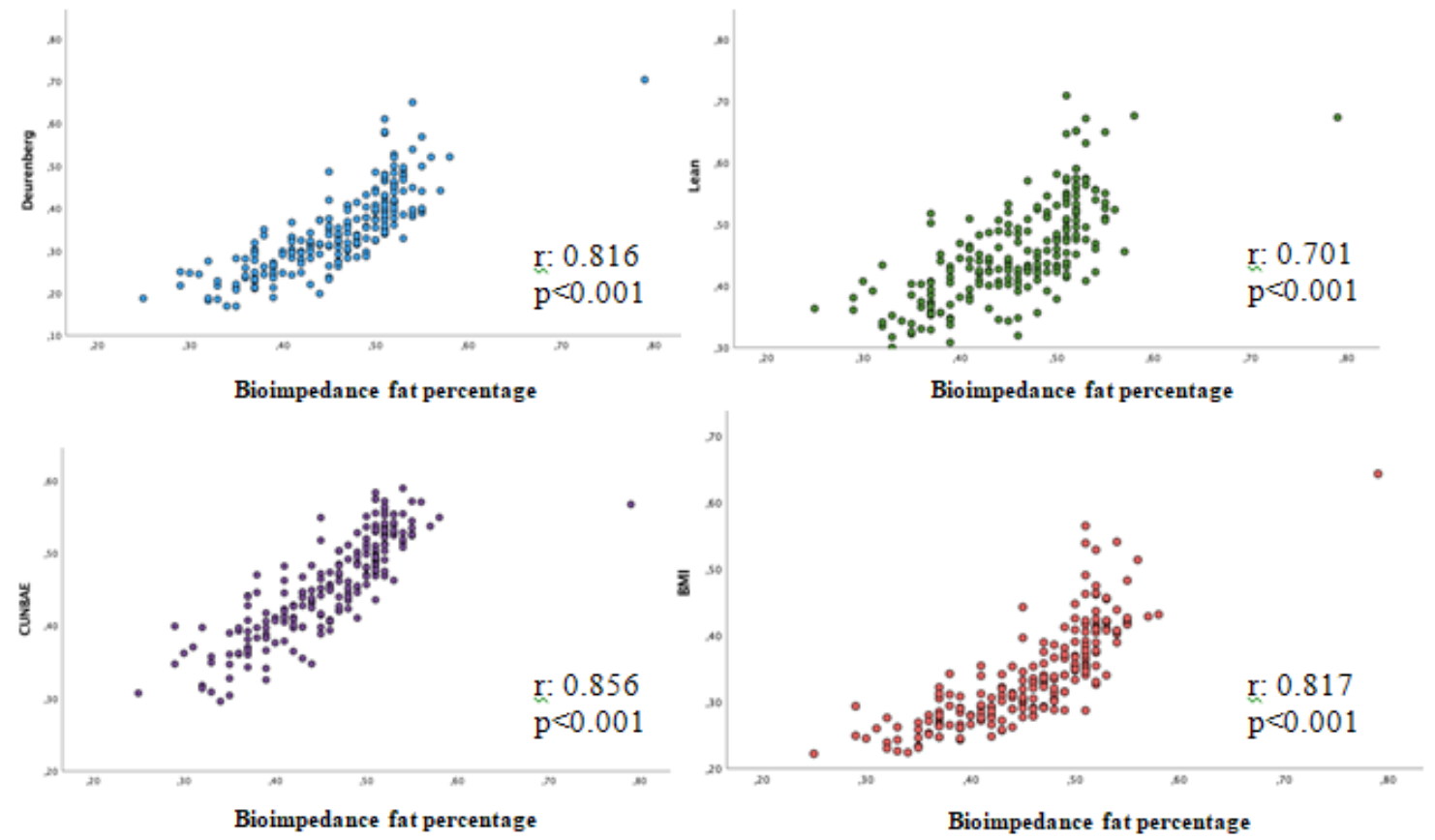

Graph 3 Correlation in women.

In the subgroup analysis (Table $3 \& 4$ ) we found that none of the formulas had statistic significance in the group of under 20 years old. For the rest of age groups in men, the three evaluated formulas had statistic significance. The group that had higher correlation was 60 years or older and 20-29 years. In women every formula had statistic significance as well, having Deurenberg's and CUN BAE formulas a higher correlation in every age group.

In the group of men from 30-39 years there's less correlation with every formal evaluated, without it meaning the loss of statistical significance. In women, the CUN BAE and Deurenberg's formula kept the same correlation in every age group.

An analysis for subgroups of body fat percentage was made, noting in both genders that the only subgroup that obtained statistic significance for the 3 formulas was the one over $40 \%$, that included the great majority of patients $(\mathrm{p}<0.001)$. We also analyzed de correlation of BMI with fat percentage. The correlation index was $0.681(\mathrm{p}<.001)$. Table 5 and 6 show the correlation index for each gender and age group. The group that has a higher correlation index was men of 60 years old or older, meanwhile the one with least correlation was men of 30-39 years. 
Table 3 Analysis by age and gender (Men)

\begin{tabular}{|c|c|c|c|c|c|c|c|c|c|c|c|}
\hline $\begin{array}{l}\text { Age } \\
\text { (n) }\end{array}$ & $\%$ Fat & Duren & Pearson & Lean & Pearson & $\begin{array}{l}\text { CUN } \\
\text { BAE }\end{array}$ & Pearson & W/H & Pearson & Average & Pearson \\
\hline $\begin{array}{l}<20 \\
(6)\end{array}$ & $\begin{array}{l}32.3- \\
40.4 \\
(35.28 \\
\text { SD 3.56) }\end{array}$ & $\begin{array}{l}37.44- \\
42.54 \\
(40.48 \text { SD } \\
2.1)\end{array}$ & $\begin{array}{l}-0.218 \\
(p=0.782)\end{array}$ & $\begin{array}{l}35.89- \\
57.34 \\
(41.98 \text { SD } \\
10.3)\end{array}$ & $\begin{array}{l}-0.597 \\
(p=0.403)\end{array}$ & $\begin{array}{l}31.66-37.1 \\
(34.9 \text { SD } \\
2.3)\end{array}$ & $\begin{array}{l}-0.239 \\
(p=0.761)\end{array}$ & $\begin{array}{l}0.63- \\
0.83 \\
(0.69 \text { SD } \\
0.08)\end{array}$ & $\begin{array}{l}-0.298 \\
(p=0.702)\end{array}$ & $\begin{array}{l}36-46 \\
(39.12 \\
\text { SD } 4.4)\end{array}$ & $\begin{array}{l}-0.54 I \\
(p=0.459)\end{array}$ \\
\hline $\begin{array}{l}20- \\
29 \\
(31)\end{array}$ & $\begin{array}{l}\text { I5.4- } \\
50.7 \\
(39.45 \\
\text { SD 9.93) }\end{array}$ & $\begin{array}{l}29.59- \\
57.35 \\
(47.3 \mathrm{SD} \\
8.1)\end{array}$ & $\begin{array}{l}0.866 \\
(p<0.00 I)\end{array}$ & $\begin{array}{l}\text { I8.76- } \\
52.56 \\
(39.73 \text { SD } \\
15.36)\end{array}$ & $\begin{array}{l}0.579 \\
(p=0.049)\end{array}$ & $\begin{array}{l}19.74- \\
47.65 \\
(39.55 \text { SD } \\
7.9)\end{array}$ & $\begin{array}{l}0.906 \\
(p<0.001)\end{array}$ & $\begin{array}{l}0.27- \\
0.82 \\
(0.65 \text { SD } \\
0.15)\end{array}$ & $\begin{array}{l}0.607 \\
(p=0.036)\end{array}$ & $\begin{array}{l}23-5 I \\
(4 I S D \\
9.27)\end{array}$ & $\begin{array}{l}0.828 \\
(p<0.00 I)\end{array}$ \\
\hline $\begin{array}{l}30- \\
39 \\
(47)\end{array}$ & $\begin{array}{l}16.6- \\
53.2 \\
(34.48 \\
\text { SD 9.47) }\end{array}$ & $\begin{array}{l}30.42- \\
86.92 \\
(48.85 \text { SD } \\
12.24)\end{array}$ & $\begin{array}{l}0.633 \\
(p<0.00 I)\end{array}$ & $\begin{array}{l}20.5- \\
76.33 \\
(41.48 \text { SD } \\
13.29)\end{array}$ & $\begin{array}{l}0.569 \\
(p=0.003)\end{array}$ & $\begin{array}{l}20.71- \\
53.56 \\
(38.34 \text { SD } \\
8.56)\end{array}$ & $\begin{array}{l}0.663 \\
(p<0.001)\end{array}$ & $\begin{array}{l}0.51- \\
I .05 \\
(0.697 \\
\text { SD 0.12) }\end{array}$ & $\begin{array}{l}0.643 \\
(p<0.001)\end{array}$ & $\begin{array}{l}24-72 \\
(42.89 \\
\text { SD } \\
12.24)\end{array}$ & $\begin{array}{l}0.629 \\
(p<0.001)\end{array}$ \\
\hline $\begin{array}{l}40- \\
49 \\
(52)\end{array}$ & $\begin{array}{l}25.7- \\
56.2 \\
(38.5 \mathrm{SD} \\
7.16)\end{array}$ & $\begin{array}{l}38.34- \\
70.71 \\
(48.56 \mathrm{SD} \\
6.99)\end{array}$ & $\begin{array}{l}0.76 \\
(p<0.001)\end{array}$ & $\begin{array}{l}29.18- \\
67.43 \\
(40.99 \mathrm{SD} \\
8.96)\end{array}$ & $\begin{array}{l}0.708\left(p^{<}\right. \\
0.001)\end{array}$ & $\begin{array}{l}28.55- \\
52.36 \\
(37.86 \text { SD } \\
5.55)\end{array}$ & $\begin{array}{l}0.786 \\
(p<0.001)\end{array}$ & $\begin{array}{l}0.54- \\
0.97 \\
(0.69 \text { SD } \\
0.096)\end{array}$ & $\begin{array}{l}0.803 \\
(p<0.00 I)\end{array}$ & $\begin{array}{l}33-64 \\
(42.47 \\
\text { SD 6.98) }\end{array}$ & $\begin{array}{l}0.764(\mathrm{p}< \\
0.001)\end{array}$ \\
\hline $\begin{array}{l}50- \\
59 \\
(39)\end{array}$ & $\begin{array}{l}23.71- \\
45.1 \\
(37.09 \\
\text { SD 5.32) }\end{array}$ & $\begin{array}{l}39.92- \\
58.88 \\
(48.25 \text { SD } \\
5.32)\end{array}$ & $\begin{array}{l}0.742 \\
(p=0.002)\end{array}$ & $\begin{array}{l}26.75- \\
60.2(40.3 \\
\text { SD } 8.16)\end{array}$ & $\begin{array}{l}0.616 \\
(p=0.019)\end{array}$ & $\begin{array}{l}28.76- \\
45.42 \\
(36.42 \text { SD } \\
4.82)\end{array}$ & $\begin{array}{l}0.754 \\
(p=0.002)\end{array}$ & $\begin{array}{l}0.56- \\
0.85 \\
(0.68 \text { SD } \\
0.07)\end{array}$ & $\begin{array}{l}0.687 \\
(p=0.007)\end{array}$ & $\begin{array}{l}32-55 \\
(41.67 \\
\text { SD 5.96) }\end{array}$ & $\begin{array}{l}0.705 \\
(p=0.005)\end{array}$ \\
\hline $\begin{array}{l}>60 \\
(33)\end{array}$ & $\begin{array}{l}22.5- \\
50.9 \\
(37,95 \\
\text { SD 8.32) }\end{array}$ & $\begin{array}{l}35.51- \\
60.94 \\
(50.18 \text { SD } \\
6.66)\end{array}$ & $\begin{array}{l}0.9(\mathrm{p} \\
<0.00 \mathrm{l})\end{array}$ & $\begin{array}{l}27.21- \\
61.21 \\
(39.47 \mathrm{SD} \\
8.94)\end{array}$ & $\begin{array}{l}0.909\left(p^{<}\right. \\
0.001)\end{array}$ & $\begin{array}{l}22.87- \\
45.56 \\
(36.70 \text { SD } \\
5.77)\end{array}$ & $\begin{array}{l}0.899 \\
(p<0.001)\end{array}$ & $\begin{array}{l}0.54- \\
0.85 \\
(0.68 \mathrm{SD} \\
0.09)\end{array}$ & $\begin{array}{l}0.953 \\
(p<0.001)\end{array}$ & $\begin{array}{l}29-56 \\
(42.15 \\
\text { SD 6.7) }\end{array}$ & $\begin{array}{l}0.954 \\
(p<0.001)\end{array}$ \\
\hline
\end{tabular}

$\mathrm{W} / \mathrm{H}$, waist/height index; Duren, durenberg formula

Table 4 Analysis by age and gender (Women)

\begin{tabular}{|c|c|c|c|c|c|c|c|c|c|c|c|}
\hline Age & $\%$ Fat & Duren & Pearson & Lean & Pearson & $\begin{array}{l}\text { CUN } \\
\text { BAE }\end{array}$ & Pearson & W/H & Pearson & Average & Pearson \\
\hline $\begin{array}{l}<20 \\
(6)\end{array}$ & $\begin{array}{l}36.6- \\
52.8 \\
(44,86 \\
\text { SD } 8.1)\end{array}$ & $\begin{array}{l}21-38 \\
(27.7 \mathrm{SD} \\
9.3)\end{array}$ & $\begin{array}{l}0.914 \\
(p=0.266)\end{array}$ & $\begin{array}{l}37-47 \\
(41.7 \mathrm{SD} \\
5.2)\end{array}$ & $\begin{array}{l}0.961 \\
(p=0.179)\end{array}$ & $\begin{array}{l}36-52 \\
(4.28 \mathrm{SD} \\
8.4)\end{array}$ & $\begin{array}{l}0.936 \\
(p=0.229)\end{array}$ & $\begin{array}{l}0.57-0.72 \\
(0.635 \mathrm{SD} \\
0.08)\end{array}$ & $\begin{array}{l}0.968 \\
(p=0.161)\end{array}$ & $\begin{array}{l}32-46(37 \\
\text { SD } 7.6)\end{array}$ & $\begin{array}{l}0.934 \\
(p=0.233)\end{array}$ \\
\hline $\begin{array}{l}20-29 \\
(31)\end{array}$ & $\begin{array}{l}29-56.3 \\
(45.24 \\
\text { SD 8.02) }\end{array}$ & $\begin{array}{l}16.86- \\
57.69 \\
(32.09 \text { SD } \\
10.65)\end{array}$ & $\begin{array}{l}0.806 \\
(p<0.00 I)\end{array}$ & $\begin{array}{l}30.81- \\
65.05 \\
(43.91 \\
\text { SD 9.35) }\end{array}$ & $\begin{array}{l}0.795 \\
(p<0.001)\end{array}$ & $\begin{array}{l}29.58- \\
57.36 \\
(44.99 \\
\text { SD 8.29) }\end{array}$ & $\begin{array}{l}0.88 \\
(p<0.001)\end{array}$ & $\begin{array}{l}0.49-0.99 \\
(0.66 \mathrm{SD} \\
0.12)\end{array}$ & $\begin{array}{l}0.803 \\
(p<0.001)\end{array}$ & $\begin{array}{l}27-60(40 \\
\text { SD 9.14) }\end{array}$ & $\begin{array}{l}0.85 \\
(p<0.001)\end{array}$ \\
\hline $\begin{array}{l}30-39 \\
(47)\end{array}$ & $\begin{array}{l}25.4- \\
56.8 \\
(43.43 \\
\text { SD 7.23) }\end{array}$ & $\begin{array}{l}|8.5|- \\
47.94 \\
(30.03 \text { SD } \\
7.47)\end{array}$ & $\begin{array}{l}0.884 \\
(p<0.00 I)\end{array}$ & $\begin{array}{l}30.03- \\
50.58 \\
(40.28 \\
\text { SD 4.8) }\end{array}$ & $\begin{array}{l}0.654 \\
(p<0.00 I)\end{array}$ & $\begin{array}{l}30.71- \\
55.46 \\
(42.83 \\
\text { SD 6.79) }\end{array}$ & $\begin{array}{l}0.899 \\
(p<0,001)\end{array}$ & $\begin{array}{l}0.45-0.77 \\
(0.59 \mathrm{SD} \\
0.07)\end{array}$ & $\begin{array}{l}0.636 \\
(p<0.001)\end{array}$ & $\begin{array}{l}26-50 \\
(37.72 \mathrm{SD} \\
6.04)\end{array}$ & $\begin{array}{l}0.875 \\
(p<0.00 I)\end{array}$ \\
\hline $\begin{array}{l}40-49 \\
(52)\end{array}$ & $\begin{array}{l}30.5- \\
79.2 \\
(45.31 \\
\text { SD 7.7) }\end{array}$ & $\begin{array}{l}20.7- \\
70.27 \\
(35.26 \mathrm{SD} \\
9.05)\end{array}$ & $\begin{array}{l}0.862 \\
(p<0.00 I)\end{array}$ & $\begin{array}{l}33.03- \\
67.27 \\
(45.48 \\
\text { SD 6.91) }\end{array}$ & $\begin{array}{l}0.715 \\
(p<0.001)\end{array}$ & $\begin{array}{l}32.74- \\
56.66 \\
(45.96 \\
\text { SD 6.I7) }\end{array}$ & $\begin{array}{l}0.771 \\
(p<0.001)\end{array}$ & $\begin{array}{l}0.47-0.97 \\
(0.64 \mathrm{SD} \\
0.1)\end{array}$ & $\begin{array}{l}0.758 \\
(p<0.001)\end{array}$ & $\begin{array}{l}31-65 \\
(42.23 \\
\text { SD 7) }\end{array}$ & $\begin{array}{l}0.833 \\
(p<0.001)\end{array}$ \\
\hline $\begin{array}{l}50-59 \\
(39)\end{array}$ & $\begin{array}{l}29.7- \\
54.4 \\
(45.65 \\
\text { SD 5.83) }\end{array}$ & $\begin{array}{l}24.7- \\
61.01 \\
(36.26 \mathrm{SD} \\
8.21)\end{array}$ & $\begin{array}{l}0.749 \\
(p<0.001)\end{array}$ & $\begin{array}{l}37.43- \\
70.8 \\
(47.78 \\
\text { SD 6.43) }\end{array}$ & $\begin{array}{l}0.648 \\
(p<0.001)\end{array}$ & $\begin{array}{l}36.19- \\
58.28 \\
(45.98 \\
\text { SD 5.79) }\end{array}$ & $\begin{array}{l}0.808 \\
(p<0.001)\end{array}$ & $\begin{array}{l}0.48-0.88 \\
(0.63 \mathrm{SD} \\
0.08)\end{array}$ & $\begin{array}{l}0.646 \\
(p<0.001)\end{array}$ & $\begin{array}{l}34-63 \\
(43.34 \mathrm{SD} \\
6.42)\end{array}$ & $\begin{array}{l}0.779 \\
(p<0.001)\end{array}$ \\
\hline $\begin{array}{l}\text { Mayor } \\
\text { o igual } \\
\text { a } 60 \\
(33)\end{array}$ & $\begin{array}{l}37.2- \\
57.9 \\
(49.02 \\
\text { SD 4.8I) }\end{array}$ & $\begin{array}{l}27.7- \\
64.93 \\
(41.63 \mathrm{SD} \\
8.89)\end{array}$ & $\begin{array}{l}0.804 \\
(p<0.001)\end{array}$ & $\begin{array}{l}40.5- \\
67.5 \\
(53.20 \\
\text { SD 6.96) }\end{array}$ & $\begin{array}{l}0.748 \\
(p<0.001)\end{array}$ & $\begin{array}{l}38.1- \\
58.88 \\
(48.51 \\
\text { SD 5.42) }\end{array}$ & $\begin{array}{l}0.868 \\
(p<0.001)\end{array}$ & $\begin{array}{l}0.01-0.93 \\
(0.86 \mathrm{SD} \\
0.15)\end{array}$ & $\begin{array}{l}0.649 \\
(p<0.001)\end{array}$ & $\begin{array}{l}35-60 \\
(47.78 \mathrm{SD} \\
6.56)\end{array}$ & $\begin{array}{l}0.866 \\
(p<0.001)\end{array}$ \\
\hline
\end{tabular}

$\mathrm{W} / \mathrm{H}$, waist/height index; Duren, deurenberg formula

Citation: Regina LS, Livia MBM,Alejandro ASPJ. Concordance between body fat percentage stablished by bio impedance and estimation formulas based in anthropometric measurements. Adv Obes Weight Manag Control. 202 I; I I (5):I59-I66. DOI: I0.I5406/aowmc.202I.II.0035 I 
Table $5 \mathrm{BMI}$ analysis by age and gender (Men)

\begin{tabular}{|c|c|c|c|}
\hline Age & $\%$ Fat & BMI & Pearson \\
\hline$<20(6)$ & $\begin{array}{l}32.3-40.4 \\
(35.28 \text { SD 3.56) }\end{array}$ & $\begin{array}{l}32-37 \\
(34.7 \mathrm{SD}\end{array}$ & $\begin{array}{l}-0.269(p 0.73 \mid) \\
\mid .8)\end{array}$ \\
\hline $20-29(3 I)$ & I5.4-50.7 (39.45 SD 9.93) & $\begin{array}{l}22-48 \\
(39.18 \mathrm{SL}\end{array}$ & $\begin{array}{l}0.894(p<0.00 I) \\
6.78)\end{array}$ \\
\hline $30-39(47)$ & 16.6-53.2 (34.48 SD 9.47) & $\begin{array}{l}24-71 \\
(38.6 \mathrm{SD}\end{array}$ & $\begin{array}{l}0.624(p<0.001) \\
10.72)\end{array}$ \\
\hline $40-49(52)$ & $25.7-56.2(38.5$ SD 7.16) & $\begin{array}{l}28-55 \\
(36.4 \mathrm{SD}\end{array}$ & $\begin{array}{l}0.785(p<0.001) \\
5.8)\end{array}$ \\
\hline $50-59(39)$ & 23.7I-45.I (37.09 SD 5.32) & $\begin{array}{l}27-44 \\
(34.26 \mathrm{SL}\end{array}$ & $\begin{array}{l}0.727(p<0.727) \\
4.5)\end{array}$ \\
\hline $\begin{array}{l}60 \text { or older } \\
-33\end{array}$ & $22.5-50.9(37,95$ SD 8.32) & $\begin{array}{l}22-43 \\
(34.17 \mathrm{SL}\end{array}$ & $\begin{array}{l}0.901(p<0.001) \\
5.53)\end{array}$ \\
\hline
\end{tabular}

\% Fat, fat percentage; BMI, body mass index

Table 6 BMI analysis by age and gender (Women)

\begin{tabular}{|c|c|c|c|}
\hline Age & $\%$ Fat & BMI & Pearson \\
\hline \multirow[t]{2}{*}{$<20(6)$} & $36.6-52.8$ & $27-42$ & $0.919(p=0.257)$ \\
\hline & $(44,86$ SD 8.1$)$ & \multicolumn{2}{|c|}{ (33.03 SD 7.7) } \\
\hline \multirow[t]{2}{*}{$20-29(3 I)$} & $29-56.3$ & $22-56$ & $0.814(p<0.001)$ \\
\hline & (45.24 SD 8.02) & \multicolumn{2}{|c|}{ (34.83 SD 8.73) } \\
\hline \multirow[t]{2}{*}{$30-39(47)$} & $25.4-56.8$ & $22-46$ & $0.880(p<0.00 I)$ \\
\hline & (43.43 SD 7.23) & \multicolumn{2}{|c|}{ (31.74 SD 6.14) } \\
\hline \multirow[t]{2}{*}{$40-49(52)$} & $30.5-79.2$ (45.3I SD 7.7) & $23-64$ & $0.863(p<0.001)$ \\
\hline & & \multicolumn{2}{|c|}{ (34.4 SD 7.53) } \\
\hline \multirow[t]{2}{*}{ 50-59 (39) } & 29.7-54.4 (45.65 SD 5.83) & $25-54$ & $0.74 I(p<0.00 I)$ \\
\hline & & \multicolumn{2}{|c|}{ (33.44 SD 6.73) } \\
\hline \multirow[t]{2}{*}{$\begin{array}{l}60 \text { or older } \\
\text { (33) }\end{array}$} & $37.2-57.9(49.02$ SD 4.8I) & $25-54$ & $0.804(p<0.00 I)$ \\
\hline & & \multicolumn{2}{|c|}{ (35.3 SD 7.135) } \\
\hline
\end{tabular}

$\%$ Fat, fat percentage; BMI, body mass index

\section{Discussion}

The results show that the anthropometric measures based formulas have a statistical significant correlation with the body fat percentage measured by bioelectrical impedance. Even though the 3 formulas had statistical significance, it is important to note that CUN BAE formula had a higher correlation index, followed by Deurenberg's formula. Lean's formula had a similar correlation to waist/height index.

In the subgroup analysis we identified that none of the formulas had statistical significance for patients under 20 years old. We assume these results are a consequence of the small number of patients within this age group. In men, CUN BAE and Deurenberg's formula have similar correlation indexes, meanwhile Lean's correlation was smaller. We think it is important to note that in the age group of men from 30-39 yeas the correlation with every formula decreases, this is note the case for women. We can identify that Deurenberg's formula tends to overestimate de body fat percentage in men in any age.
In women, the CUN BAE formula had higher correlation indexes, nonetheless, Deurenberg's and Lean's formulated similar values in every age group. Another difference between the formulas evaluated in women and men, is that Deurenberg's formula underestimates body fat percentage in women of all ages.

We also conducted a subgroup analysis according to body fat percentage with statistical significance only in the groupr over $40 \%$ of body fat. We are certain that these results are a consequence of the small number of participants within the rest of the groups. Duerenberg's formula has the most disperse results.

Our findings do not correlate with the ones made by Al-Gindan et al. They found that there was a greater correlation in men with Lean's formula and in women with Deurenberg's formula. Those differences could be explained by the fact that Al-Gindan's studied population had normal BMI and not only one ethnic group. However, the findings we had with CUN BAE formula correlate with various trials done by Marrero-Gordillo (2015), Gómez-Marcos (2019), and Molano-Tovar (2021) that show a strong correlation between the formula and body fat percentage.

Our trial is the first conducted in Latin America with the objective to evaluate the correlation of the formulas in patients with obesity and overweight, so it can be used for further investigations in order to calculate sensibility an specificity of each formula for the diagnosis of obesity in our population. Besides, it is the first study to compare the 3 formulas, so its findings can be useful in order to individualice the use of each.

Some of the disadvantages of our study are that the majority of our population has a body fat percentage $<40 \%$, so we could not have statistical significance in the rest of the groups. We also had few participants under the age of 20 , so our results can't be extended to that age group. Also, our study was retroactive, so some of our data could be biased by mistakes in registry. Finally, it is important to assess this formulas in healthy individuals.

The strong correlation between body fat porcentage measured either by impedance or calculated by this formulas shown in this study, offers a reliabil tool for obesity diagnosis in the clinic enviroment, specially when bioimpedance is not available, considering the limitations that BMI has for the diagnosis.

\section{Limitations}

Our results cannot be generalized to healthy population, or to other ethnic groups, and the obtained results cannot be used to calculate neither sensibility nor specificity because our population did not have normal weight individuals. The correlation is limited con the fat mass porcentage. Another limitation of the study was the small number of patients with a BMI grater than $40 \mathrm{~kg} / \mathrm{m}^{2}$.

\section{Conclusion}

The anthropometric based formulas have correlation with de body fat percentage measured by bioimpedance, so they can be used in Latin population with obesity in order to estimate fat percentage. CUN BAE and Deruenberg's formulas have a greater correlation in men, meanwhile CUN BAE formula had a greater correlation in women.

\section{Ethic considerations}

We didn't conducted any kind of intervention and the obtained data were only used by the authors of this paper. The researchers that conducted this trial have no conflicts of interest. 


\section{Acknowledgments}

None.

\section{Conflicts of interest}

We disclose no conflicts of interest in this study.

\section{Funding}

None.

\section{References}

1. National Health and Nutrition Survey. In: INEGI, editor. Mexico: Instituto Nacional de Salud Pública; 2018.

2. Front tag: a theme of child health protection: Panamerican Health Organization. 2019.

3. Bluher M. Obesity: global epidemiology and pathogenesis. Nat Rev Endocrinol. 2019;15(5):288-298.

4. Andreoli A, Garaci F, Cafarelli FP, et al. Body composition in clinical practice. Eur J Radiol. 2016;85(8):1461-1468.

5. Deurenberg P, J A Weststrate, J C Seidell. Body mass index as a measure of body fatness: age- and sex- specific prediction formulas. British Journal of Nutrition. 1991;65:105-114.

6. Collaboration NCDRF. Trends in adult body-mass index in 200 countries from 1975 to 2014: a pooled analysis of 1698 populationbased measurement studies with 19.2 million participants. Lancet. 2016;387(10026):1377-1396.

7. Berrington de Gonzalez A, Hartge P, Cerhan JR, et al. Body-mass index and mortality among 1.46 million white adults. $N$ Engl J Med. 2010;363(23):2211-2219.

8. Longo M, Zatterale F, Naderi J, et al. Adipose Tissue Dysfunction as Determinant of Obesity-Associated Metabolic Complications. Int J Mol Sci. 2019;20(9).

9. Porter SA, Massaro JM, Hoffmann U, et al. Abdominal subcutaneous adipose tissue: a protective fat depot? Diabetes Care. 2009;32(6):10681075.

10. Lee SJ, Shin SW. Mechanisms, Pathophysiology, and Management of Obesity. N Engl J Med. 2017;376(15):1491-1492.

11. Hopkins M, Blundell JE. Energy balance, body composition, sedentariness and appetite regulation: pathways to obesity. Clin Sci (Lond). 2016;130(18):1615-1628.

12. Forbes GB. Human body composition: growth, aging, nutrition, and activity. New York: Springer - Verlag; 2012. 343 p.

13. Ross R, Soni S, Houle SA. Negative Energy Balance Induced by Exercise or Diet: Effects on Visceral Adipose Tissue and Liver Fat. Nutrients. 2020;12(4).

14. Nuttall FQ. Body Mass Index: Obesity, BMI, and Health: A Critical Review. Nutr Today. 2015;50(3):117-28.

15. Keys A FF, Karvonen M, Kimura N, et al. Indices of relative weight and obesity. Journal of Chronic Diseases. 1972;25(6-7):329-343.

16. Romero-Corral A, Somers VK, Sierra-Johnson J, et al. Accuracy of body mass index in diagnosing obesity in the adult general population. Int $J$ Obes (Lond). 2008;32(6):959-966.

17. Mazzoccoli G. Body composition: Where and when. Eur J Radiol. 2016;85(8):1456-1460.
18. Stefan N, Kantartzis K, Machann J, et al. Identification and characterization of metabolically benign obesity in humans. Arch Intern Med. 2008;168(15):1609-1616.

19. Oliveros E, Somers VK, Sochor O, et al. The concept of normal weight obesity. Prog Cardiovasc Dis. 2014;56(4):426-433.

20. Thibault R, Pichard C. The evaluation of body composition: a useful tool for clinical practice. Ann Nutr Metab. 2012;60(1):6-16.

21. Teigen LM, Kuchnia AJ, Mourtzakis M, et al. The Use of Technology for Estimating Body Composition Strengths and Weaknesses of Common Modalities in a Clinical Setting. Nutr Clin Pract. 2017;32(1):20-29.

22. Pineau JC, Frey A. Comparison of skinfold thickness models with DEXA: impact of visceral adipose tissue. J Sports Med Phys Fitness. 2016;56(5):541-545.

23. Kuriyan R. Body composition techniques. Indian $J$ Med Res. 2018;148(5):648-658.

24. Shepherd JA, Ng BK, Sommer MJ, Heymsfield SB. Body composition by DXA. Bone. 2017;104:101-105.

25. Achamrah Najate, Colange Guillaume, Delay Julie, et al. Comparison of body composition assessment by DXA and BIA according to the body mass index: A retrospective study on 3655 measures. PLOS ONE. 2018;13(7).

26. Fedewa MV, Nickerson BS, Esco MR. Associations of body adiposity index, waist circumference, and body mass index in young adults. Clin Nutr. 2019;38(2):715-720.

27. Nickerson BS, Esco MR, Fedewa MV, et al. Development of a Body Mass Index-based Body Fat Equation: Effect of Handgrip Strength. Med Sci Sports Exerc. 2020;52(11):2459-2465.

28. Deurenberg P. Body mass index as a measure of body fatness: ageand sex- specific prediction formulas. British Journal of Nutrition. 1991;65:105-114.

29. Lean ME, Han TS, Deurenberg P. Predicting body composition by densitometry from simple anthropometric measurements. Am J Clin Nutr. 1996;63(1):4-14.

30. Gomez-Ambrosi J, Silva C, Catalan V, et al. Clinical usefulness of a new equation for estimating body fat. Diabetes Care. 2012;35(2):383-388.

31. Vinknes KJ, Nurk E, Tell GS, et al. The relation of CUN-BAE index and BMI with body fat, cardiovascular events and diabetes during a 6-year follow-up: the Hordaland Health Study. Clin Epidemiol. 2017;9:555-566.

32. Sanchez E, Sanchez M, Betriu A, et al. Are Obesity Indices Useful for Detecting Subclinical Atheromatosis in a Middle-Aged Population? Obes Facts. 2020;13(1):29-39.

33. Al-Gindan YY, Hankey CR, Govan L, et al. Derivation and validation of simple anthropometric equations to predict adipose tissue mass and total fat mass with MRI as the reference method. Br J Nutr. 2015;114(11):18521867.

34. Fernández JR, Moonseong $\mathrm{H}$, Heymsfield $\mathrm{S}$, et al. Is percentage body fat differentially related to body mass index in Hispanic Americans, African Americans, and European Americans?. Am J Clin Nut. 2003;77(1): 71-75.

35. Casas YG, Schiller BC, DeSouza CA, et al. Total and regional body composition across age in healthy Hispanic and white women of similar socioeconomic status. Am J Clin Nutr. 2001;73(1):13-18.

36. Wong WW, Strizich G, Heo M, et al. Relationship between body fat and BMI in a US hispanic population-based cohort study: Results from HCHS/SOL. Obesity (Silver Spring). 2016;24(7):1561-1571. 